\title{
Percepções de Alunos de Medicina sobre Marketing Médico
}

\section{Undergraduate Medical Student's Perceptions Regarding Professional Marketing}

\author{
Fernando Henrique Canhoto Alves ${ }^{I}$, Fernanda Pedrosa Torres ${ }^{I}$, Hilda Satie Suto ${ }^{I}$ \\ Lunia Sofia Lima AzevedoI, Marcell Maduro Barbosa ${ }^{I}$, Renato Martins Pedro \\ Ana Carolina Delazia Albuquerque Santana ${ }^{I}$, Daniella Rantin ${ }^{I}$, Fábio Henrique Luiz Leonardo \\ Marina Gomes de AndradeI, Natasha Nicos FerreiraI , Antonio Pazin Filho
}

\author{
PALAVRAS-CHAVE \\ - Marketing de Serviços de Saúde. \\ - Ética Profissional. \\ - Educação Médica. \\ - Tutoria.
}

\author{
KEYWORDS \\ - Marketing of Health Services. \\ - Professional Ethics. \\ - Medical Education. \\ - Preceptorship.
}

\section{Recebido em: 27/03/2011}

Reencaminhado em: 23/10/2011

Aprovado em: 21/05/2012

\section{RESUMO}

Introdução: Marketing médico é um assunto controverso, principalmente no que concerne a princípios éticos. Portanto, frente à competição acirrada de mercado, é necessário o preparo profissional. Conhecer a percepção dos alunos de Medicina pode auxiliar na estruturação de alternativas de capacitação. Metodologia: Inicialmente, identificaram-se crenças sobre marketing médico através de grupo focal composto por 12 alunos. Com base nesses dados, dez afirmações para avaliar atitudes foram aplicadas aos alunos de uma Faculdade de Medicina pública brasileira. Resultados: Observou-se falta de clareza sobre o conceito de marketing, preocupação com princípios éticos e necessidade de marketing no mercado competitivo. $\mathrm{Na}$ fase de aplicação, foram obtidas 280 respostas de diversos estágios do curso. Apenas 16,8\% admitiram contato com o tema. Houve clareza sobre ética em relação ao paciente, influenciada positivamente pela progressão no curso, mas houve divergência na ética entre profissionais. Conclusões: Marketing médico é uma área pouco compreendida e relegada ao currículo oculto, sendo influenciada por transposições inadequadas de métodos didáticos destinados à comunicação profissional para a população leiga. Novos métodos de ensino, como a educação tutorial, podem ser uma alternativa para lidar com essas situações.

\section{ABSTRACT}

Background: Professional marketing is a controversial topic, mainly regarding ethical principles. Therefore, increasing market competion turns professional capacitation mandatory. To know under graduation medical student's perceptions could help to develop educational alternatives to face this problem. Metodology: First, professional marketing believes were identified through focus group methodology including twelve students. Based on these results, ten affirmatives were constructed to evaluate attitudes and applied to the students of a Brazilian public medicine school. Results: It was observed lack of a clear definition of marketing, preoccupation regarding ethical principles and need to employ marketing strategies in a competitive market. At the survey stage, 280 answers from different stages of the course were obtained. Only $16.8 \%$ admitted to have had previous contact with the topic. Clear ethical standards for medical-patient relationship were determined, increasing with course progression. However inter-professional ethical principles was more subject to confusion. Conclusion: Professional marketing is poorly understood and it is relegated to the occult curricula, being influenced by didactic methods inadequately transposed for marketing strategies. Newer educational methods, like mentoring, could be an alternative to deal with these situations. 


\section{INTRODUÇÃO}

A formação médica é extremamente discutida, sendo senso comum que a duração preconizada de seis anos se torna cada vez mais insuficiente frente aos avanços do conhecimento ${ }^{1,2}$. Esse enorme conteúdo exerce pressão constante sobre os alunos, que naturalmente se dedicam a absorvê-lo às custas do tempo que deveriam dedicar à aquisição de habilidades práticas ou atitudes ético-profissionais ${ }^{3,4}$. Somado a isso, temos enfrentado a abertura desenfreada de novas escolas médicas, sem o proporcional aumento de programas de residência médica para especialização posterior à formação ${ }^{5}$. Como o acesso aos programas de residência médica está em muito pautado no conhecimento teórico demonstrado pelo candidato na avaliação, esse é mais um fator que direciona o aluno para adquiri-lo em detrimento das habilidades e atitudes necessárias ${ }^{6,7}$.

O problema é ainda mais complexo em razão da falta de consenso sobre as habilidades e atitudes que devem ser adquiridas $^{8}$. O aprendizado de habilidades e atitudes é pautado em muito pelo exemplo, fazendo com que, mesmo que este aprendizado esteja previsto no currículo formal, o currículo informal e o currículo oculto passem a exercer influência significativa9. Uma dessas atitudes compreende o marketing médico, que representa um conjunto de ações e estratégias que tem por objetivo agregar valor à prática médica por meio da identificação de oportunidades e mercado, dos desejos e das necessidades dos pacientes ${ }^{10-12}$. O marketing médico é motivo de elevada controvérsia, sendo considerado por muitos como um processo que fere os princípios éticos que regem a medicina e excluído do currículo formal. No entanto, frente à competição acirrada de mercado, trata-se de uma realidade para a qual as instituições formadoras devem capacitar seus alunos.

O Programa de Educação Tutorial (PET) da Secretaria de Ensino Superior do Ministério da Educação é composto por grupos de estudantes de cursos de graduação das Instituições de Ensino Superior (IES) do País que desenvolvem atividades buscando indissociabilidade entre ensino, pesquisa e extensão sob a tutoria de um docente. No contexto da Faculdade de Medicina de Ribeirão Preto da Universidade de São Paulo (FMRP-USP), o PET está vinculado à Comissão de Graduação, desenvolvendo diversas atividades de caráter coletivo, sendo um dos enfoques o ensino médico (http://www.fmrp.usp.br/ pet/). Como parte de suas atividades em 2010, o PET-FMRP-USP realizou um simpósio temático sobre marketing médico, convidando autoridades do Conselho Regional de Medicina do Estado de São Paulo (Cremesp), profissionais médicos e administradores para uma discussão aberta à comunidade.
Previamente a este simpósio, buscou-se conhecer o conceito dos estudantes de Medicina da FMRP-USP sobre Marketing Médico, de modo a prover material para as palestras.

O objetivo deste trabalho é apresentar as percepções de estudantes de Medicina sobre marketing médico, contextualizando essas percepções à formação, de modo a contribuir para a estruturação curricular.

\section{METODOLOGIA}

Para avaliar as percepções dos alunos sobre marketing médico, foi desenvolvido um instrumento, constituído pelas afirmações da Tabela 1. Os alunos deveriam responder se consideravam cada afirmação verdadeira ou falsa. Esse instrumento foi desenvolvido em etapas. Inicialmente, utilizou-se metodologia qualitativa de grupo focal, constituído pelos próprios integrantes do grupo (5 alunos do segundo ano; 4 alunos do terceiro; e 3 alunos do quarto ano), para se obterem ideias, dúvidas e crenças sobre marketing médico ${ }^{13}$. Posteriormente, frente aos dados obtidos, foi realizada uma pesquisa bibliográfica, a fim de aprofundar o conhecimento sobre os temas identificados. Com base nas fontes localizadas, foram construídas situações fictícias sobre marketing médico para avaliar atitude frente ao problema, em vez de se explorar conhecimento teórico sobre marketing médico. Essas situações compuseram as afirmações do instrumento aplicado aos alunos do primeiro ao sexto ano do curso médico, após ter sido aprovado pela Comissão de Ética em Pesquisa do Hospital das Clínicas da FMRP-USP e pela Comissão de Graduação da FMRP-USP.

As questões de 1 a 6 foram embasadas na Resolução CFM no $1.701 / 2003^{14}$, as questões de 7 a 9 na referência 11 , e a última questão solicitava a exposição ao conceito de marketing médico durante a formação. A participação foi voluntária, e os participantes foram informados de que o resultado seria divulgado no simpósio temático e no órgão de divulgação do grupo PET (revista Vedas), preservando-se o anonimato individual.

O instrumento foi aplicado antes do simpósio temático, o qual foi constituído de uma parte expositiva, incluindo conceitos sobre marketing médico na visão de um administrador especializado no assunto e do Cremesp. A segunda parte foi constituída por mesa-redonda composta por dois profissionais médicos, um administrador, um conselheiro do Cremesp, um professor de ética médica e um acadêmico do grupo PET, que debateram as respostas às afirmações. $\mathrm{O}$ debate foi aberto aos 105 participantes da plateia, sendo as considerações anotadas para fomentar a presente discussão.

As respostas obtidas foram agrupadas nas três fases de formação (básico; clínico; internato) do currículo da FMRP- 


\section{TABELA 1}

Número absoluto e porcentagem (entre parênteses) das respostas consideradas verdadeiras para as afirmações contidas no instrumento apresentado aos alunos de medicina da FMRP-USP de acordo com o ciclo básico, clínico ou internato. A resposta correta é apresentada na última coluna da tabela.

\begin{tabular}{|c|c|c|c|c|c|c|c|}
\hline 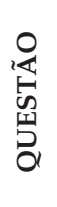 & AFIRMAÇÃO & 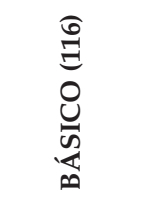 & 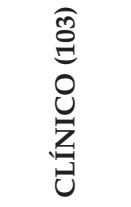 & 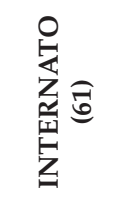 & 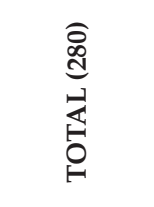 & $\mathbf{P}$ & 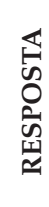 \\
\hline 1 & $\begin{array}{l}\text { A internet é uma ótima ferramenta para divulgação de novos } \\
\text { tratamentos realizados exclusivamente por uma clínica ou grupo de } \\
\text { profissionais médicos. }\end{array}$ & $80(68,9)$ & $55(54,4)$ & $33(54,1)$ & $168(60,40)$ & 0,04 & $\mathrm{~F}^{15}$ \\
\hline 2 & $\begin{array}{l}\text { Fazer palestras educativas para a comunidade se constitui em forma } \\
\text { de propaganda válida }\end{array}$ & $99(86,0)$ & $89(86,4)$ & $52(85,2)$ & $240(86,0)$ & 0,97 & $\mathrm{~V}^{15}$ \\
\hline 3 & $\begin{array}{l}\text { Um procedimento de ponta recém-disponibilizado no exterior está } \\
\text { sendo validado em um projeto de pesquisa. A propaganda de uma } \\
\text { clínica inclui a disponibilidade desse equipamento. Isto é válido, já } \\
\text { que se trata de procedimento que está sendo estudado. }\end{array}$ & $21(18,4)$ & $9(8,7)$ & $5(8,2)$ & $35(12,6)$ & 0,05 & $\mathrm{~F}^{15}$ \\
\hline 4 & $\begin{array}{l}\text { É lícito incluir imagens de pacientes antes e após tratamento em } \\
\text { folhetos explicativos de clínicas, desde que se obtenha a autorização } \\
\text { do paciente. }\end{array}$ & $89(77,4)$ & $68(66,6)$ & $47(77,0)$ & $204(77,0)$ & 0,15 & $\mathrm{~F}^{15}$ \\
\hline 5 & $\begin{array}{l}\text { Um colega foi eleito “Médico do Ano" por uma associação } \\
\text { beneficente. É lícito utilizar esta premiação em propaganda. }\end{array}$ & $49(43,3)$ & $50(49,0)$ & $39(63,9)$ & $138(50,0)$ & 0,03 & $\mathrm{~F}^{15}$ \\
\hline 6 & $\begin{array}{l}\text { O site pessoal de um médico disponibiliza a filmagem de um } \\
\text { procedimento cirúrgico, ocultando quaisquer detalhes que permitam a } \\
\text { identificação do paciente. Como se trata de ilustração de um } \\
\text { procedimento técnico, realizada com fins didáticos, isto é uma forma } \\
\text { de propaganda válida. }\end{array}$ & $70(60,8)$ & $59(57,8)$ & $40(66,6)$ & $169(61,0)$ & 0,53 & $\mathrm{~F}^{15}$ \\
\hline 7 & $\begin{array}{l}\text { Estruturação de consultório (mobília e decoração) e treinamento de } \\
\text { equipe auxiliar (secretárias) são meios eficazes de marketing médico. }\end{array}$ & $102(87,9)$ & $94(91,2)$ & $59(98,3)$ & $255(98,3)$ & 0,06 & $\mathrm{~V}^{11}$ \\
\hline 8 & $\begin{array}{l}\text { O marketing não serve para divulgar o médico, mas sim para oferecer } \\
\text { qualidade ao paciente. }\end{array}$ & $40(34,5)$ & $33(32,0)$ & $14(22,9)$ & $87(31,0)$ & 0,27 & $\mathrm{~V}^{11}$ \\
\hline 9 & $\begin{array}{l}\text { Encarar o paciente como cliente é a base do marketing médico, mas } \\
\text { isto pode ocasionar falha da confiança do paciente no profissional. }\end{array}$ & $88(76,5)$ & $73(71,5)$ & $32(52,4)$ & $193(69,4)$ & 0,004 & $\mathrm{~F}^{11}$ \\
\hline 10 & $\begin{array}{l}\text { Eu já tive noções sobre marketing médico na faculdade, quer na forma } \\
\text { de cursos formais, quer em discussões com professores ou por } \\
\text { atividades extra-curriculares. }\end{array}$ & $7(6,0)$ & $23(22,3)$ & $17(27,8)$ & $47(16,8)$ & 0,00 & \\
\hline
\end{tabular}

* questões em branco que foram desconsideradas na análise - Q1(2); Q2(1); Q3(2); Q4(2); Q5(2);Q6(3); Q7(1); Q9(1).

-USP, para avaliar o impacto da evolução do curso sobre o tema. As variáveis categóricas foram expressas em porcentagem, utilizando-se o teste do Qui-Quadrado para a diferença entre grupos. As respostas em branco foram desconsideradas na análise. Para todos os testes utilizados, considerou-se significância estatística o valor de $p<0,05$. A análise dos dados foi realizada com o programa Stata versão $10^{15}$.

\section{RESULTADOS}

$\mathrm{Na}$ fase de elaboração do instrumento, identificaram-se como fatores preestabelecidos ou que geravam curiosidade ou preocupação no grupo: falta de clareza sobre o conceito de marketing; preocupação em ferir algum princípio ético ao realizar propaganda; necessidade de realizar marketing no mercado de trabalho competitivo; o currículo do profissional como 
meio de divulgação; novos meios de divulgação de informação e forma de utilização; possibilidade de transpor conceitos de outras áreas para a medicina e problemas nesse processo. Com base nesses dados, foram identificadas como possíveis fontes para elucidar as perguntas geradas duas referências principais, que sanaram parcialmente as dúvidas e embasaram a formulação do instrumento de pesquisa ${ }^{11,15}$

Foram obtidas $280(46,7 \%)$ respostas dos 600 alunos matriculados na FMRP-USP, sendo 116 (41,4\%) do ciclo básico, 103 (36,8\%) do ciclo clínico e 61 (21,8\%) do internato. O instrumento foi aplicado previamente ao seminário temático, sendo que nenhum outro questionário foi aplicado após a sua realização. No geral, o tema era desconhecido por uma grande maioria dos entrevistados (apenas 16,8\% dos alunos já haviam tido contato com o tema de alguma forma durante o curso). Os resultados obtidos são apresentados na Tabela 1.

Houve variação entre $12,6 \%$ e $98,3 \%$ na porcentagem total de acerto de cada questão. Nas perguntas embasadas na Resolução CFM no 1.701/2003, a questão 3 foi a que teve menor taxa de acerto, com proporção decrescente do básico para o internato. As questões 1 e 5 tiveram comportamento oposto. As questões 2, 4 e 6 não foram discriminatórias. Nas questões de conceito sobre marketing médico, apenas a questão 9 foi discriminatória, apresentando redução da proporção de acerto do básico para o internato.

\section{DISCUSSÃO}

Cerca de 4,7\% dos processos abertos no Cremesp se referem à publicidade médica ilegal, embora $85 \%$ das denúncias sejam desconsideradas, pois o médico tem a oportunidade de se redimir, assinando termo de compromisso de não incorrer novamente na infração. Na grande maioria dos casos, o médico desconhece a impropriedade de suas ações. No presente trabalho, apenas $16,8 \%$ dos alunos tiveram contato com o tema, atingindo $27,8 \%$ entre os alunos do internato, refletindo o fato de não haver curso curricular formal, embora deva estar sendo abordado no currículo oculto. Esses dados mostram a importância do assunto e da discussão sobre a melhor forma de inserir este e outros temas similares na grade curricular.

O conhecimento incompleto sobre marketing médico, já presente na fase de elaboração, se reproduziu na fase de aplicação. Para a maioria dos entrevistados, o marketing só diz respeito à projeção do médico e não ao fato de agregar valor ao paciente (questão 8). Isto pode ser congruente com o fato de a grande maioria dos alunos considerar a estruturação do consultório (questão 7) como estratégia de marketing válida, pois isto pode ser compreendido como propaganda para o médico e não como valor para o paciente. Marketing implica um processo de ganha-ganha, ou seja, o médico angaria novos pacientes à medida que estes se sintam valorizados ${ }^{11}$. Para tanto, o processo deve incluir valores do paciente, como, por exemplo, ser bem atendido por recepcionistas, esperar num ambiente agradável ou mesmo receber uma ligação de acompanhamento do médico. Quando se pensa em marketing, a primeira impressão que vem à mente é a propaganda em meios de comunicação, o que não agrega valor ao atendimento do paciente e pode implicar infrações éticas. Compreender o conceito de marketing pode evitar erros e possibilitar o alcance dos verdadeiros objetivos ${ }^{11,15}$.

$\mathrm{O}$ receio de infringir preceitos éticos na relação médico-paciente foi extensamente discutido na fase de elaboração. As respostas à questão 9 mostraram redução desse receio com a progressão no curso, mas esse sentimento ainda foi considerável no internato. Os padrões encontrados para o modus faciendi do marketing (questões 1 a 6) são contraditórios. Por um lado, as respostas às questões 2 e 3 sugerem cuidado com o paciente, buscando educá-lo e protegê-lo de procedimentos experimentais, que, se não estão presentes no ciclo básico, são enfatizados ao longo do curso. Isto pode ser compreendido como reflexo da ênfase em princípios éticos na relação médico-paciente presente ao longo de todo o curso médico. No entanto, as respostas às questões 1,4 e 6 expõem certa confusão para algumas situações importantes que expõem princípios de relação interprofissional indireta, ou seja, por meio de propaganda, que, embora devam ser também pautadas em princípios éticos, não são tão enfatizadas como na relação médico-paciente. Para essas questões, o impacto da progressão do curso não foi significativo.

Um dos pontos que gerava preocupação ou dúvida na fase de elaboração foi a utilização do currículo profissional como base para marketing. A questão 5 explorou esse conceito, sendo considerada verdadeira pela maioria dos entrevistados, embora vetada pelo Conselho Federal de Medicina Resolução CFM nº 1.701/2003 Art. 12. A base para essa resolução é a preocupação com concorrência desleal entre os médicos, pois títulos dessa natureza podem ser obtidos por outras vias além do mérito profissional, muitas vezes refletindo a projeção social do médico e não sua competência técnica. A preocupação com o desenvolvimento de um currículo competitivo é grande entre estudantes de Medicina, principalmente pela disputa de uma vaga de residência. As IES devem se preocupar em instruir seus alunos sobre a correta elaboração desses instrumentos, deixando clara sua finalidade. Uma possível interpretação para as respostas obtidas à questão 5 é a transposição inadequada da elaboração de um currículo 
destinado a fins profissionais para marketing entre a população leiga.

A utilização de novos meios de divulgação, principalmente na rede mundial de computadores, também gerou dúvida na fase de elaboração. Os profissionais médicos estão sendo aconselhados por especialistas em marketing a desenvolver uma página na rede mundial, acrescentando informações para potenciais pacientes. No entanto, esse meio de divulgação deve ser pautado pelos mesmos princípios éticos aplicáveis a outros meios. A questão 1 explora esse tópico ao expor a vantagem competitiva que um grupo pode ter sobre potenciais concorrentes por realizar um procedimento exclusivo. Isto é vetado pela Resolução CFM no 1.701/2003, Art. 9, Par. 1, itens b e c, pois o médico não pode realizar concorrência desleal. Um grupo que tem condições de adquirir uma página na rede mundial de computadores ou alegar exclusividade sobre um novo recurso passa a ter uma vantagem competitiva sobre os que não têm, caracterizando concorrência desleal.

Outro aspecto a ressaltar é como veicular a informação para a população leiga. A questão 4 propõe a utilização de fotos que ilustrem situações antes-depois. A Resolução CFM no $1.701 / 2003$, Art. $3^{\circ}$, item g proíbe especificamente esse tipo de estratégia, extremamente comum. Isto se deve ao fato de o médico não poder garantir o resultado, em virtude da reação individual do paciente e de possíveis complicações. A exposição de situações antes-depois passa a mensagem de que o resultado será obtido, constituindo propaganda enganosa. O fato de se obter o consentimento do paciente para realizar a divulgação não evita que potenciais problemas ocorram com outros pacientes, embora esse consentimento tranquilize o médico e desvie a reflexão sobre ética profissional para ética com o paciente.

No mesmo sentido, a demonstração de procedimentos cirúrgicos ou de quaisquer outros procedimentos em detalhes que devem ser reservados ao ambiente profissional não deve ser transposta para a população leiga, podendo ferir a sensibilidade de pessoas que não estão acostumadas a esse tipo de demonstração (questão 6). Além disso, muitas vezes, essa demonstração é desnecessária para se explicar o procedimento em linguagem compreensível pelo paciente. Vale ressaltar que, no processo de educação médica, essas estratégias são utilizadas em aulas expositivas para demonstração de procedimentos, tanto em situações de graduação, como em eventos de educação médica continuada ${ }^{16-18}$. Embora apropriada ao ambiente profissional, a transposição dessa estratégia para meios de divulgação é inadequada.

Finalmente, a questão 9 foi o ponto mais controverso entre os debatedores no simpósio. O conceito de paciente como cliente, embora comum em administração, não é bem aceito pelo médico. Esse tema está sendo motivo de grandes debates, principalmente por motivar o uso do código de defesa do consumidor para analisar procedimentos médicos. Embora a resposta tenha sido considerada correta pela referência 11, que lhe serviu de base, não pode ser extrapolada como asserção definitiva, haja vista que ainda é motivo de debate na sociedade.

Uma consideração importante é que esse trabalho está embasado na Resolução CFM no 1.701/2003. Recentemente, foi publicada a Resolução CFM nº 1.974/2011, que atualiza e expande os conceitos descritos na resolução anterior. Vale a ressalva de que todos os princípios descritos nesse trabalho foram mantidos na nova resolução, e os aspectos sobre propaganda via rede mundial de computadores receberam especial ênfase, bem como a proibição de assistência médica a distância (via rede mundial ou telefone), a vedação de anúncios de determinados títulos e certificados, e a extensão das regras a instituições, como sindicatos e sociedades médicas.

O ensino de habilidades e atitudes é um verdadeiro desafio frente à inadequação de métodos convencionais ${ }^{17}$. Novos métodos, como a simulação, estão ganhando forma em laboratórios de habilidades instalados em diversas IES, mas a utilização desses laboratórios está voltada principalmente para a aquisição de habilidades mais concretas ${ }^{19,20}$. Atitudes como as que se espera que o aluno tenha frente ao marketing médico são fruto de atividade reflexiva. Embora possa ser adquirido por métodos como simulação e ensino baseado em problemas, isto requer atividades específicas, e pode não haver carga horária para contemplar todas as atitudes que venham a surgir. Marketing médico é um exemplo de uma nova realidade que requer comportamento ético do profissional que não existia com a ênfase observada nos últimos cinco anos. Para lidar com essas situações, o ensino tutorial pode ser mais adequado, por ser adaptável às necessidades do grupo. Esse método possibilita reflexão para a identificação, elaboração e difusão de conhecimento que não está sendo abordado no currículo convencional, sob adequada supervisão. Mais do que tudo, tem o potencial de desenvolver no aluno a capacidade de enfrentar novos problemas no futuro com metodologia similar.

Foi impossível conseguir que todos os alunos da FMRP-USP respondessem ao questionário. O evento foi realizado no final do ano letivo e aplicado num intervalo máximo de duas semanas antes do simpósio temático, para motivar a participação da comunidade estudantil. Esse curto intervalo, somado ao fato de os alunos do clínico e do internato se encontrarem dispersos em múltiplos estágios e já em fase final de curso, 
contribuiu para a menor taxa de respostas nesses grupos. Embora esses fatores nos obrigassem a utilizar uma amostragem de conveniência e certamente constituam uma limitação, não há motivos para supor que os alunos que não participaram tenham sido expostos a um aprendizado sobre marketing médico diferenciado, haja vista que não há curso curricular formal ou curso extracurricular oferecido pela FMRP-USP. Obter dados após a realização do simpósio seria incluir possível viés, já que os alunos que não participaram da pesquisa poderiam ter acesso por contiguidade ao que foi discutido no evento, sendo por isso evitado.

Em resumo, marketing médico é uma área pouco compreendida e não abordada no currículo formal da FMRP-USP e de muitas outras IES. Trata-se de um exemplo típico de tema relegado ao currículo oculto, sendo por isso mesmo influenciado por transposições inadequadas de métodos didáticos destinados à comunicação profissional para a comunicação com a população leiga. A preocupação em desenvolver bases éticas sólidas na relação interprofissional deve ser a mesma empregada na relação médico-paciente. Novos métodos de ensino, como a educação tutorial, podem representar uma alternativa para lidar com essas situações na formação profissional.

Apoio: Esta pesquisa foi realizada com verba dedicada pelo Programa de Educação Tutorial da Secretaria de Educação Superior do Ministério da Educação. Os alunos e o tutor recebem bolsa regulamentada pelo programa. O simpósio temático sobre Marketing Médico foi realizado com esse fomento e com recursos doados pela Associação Paulista de Medicina e Fundação de Amparo à Pesquisa, Ensino e Assistência (Faepa) do Hospital das Clínicas da Faculdade de Medicina de Ribeirão Preto da Universidade de São Paulo (HC-FMRP-USP)

\section{AGRADECIMENTOS}

Os autores agradecem a Isac Jorge Filho, conselheiro do Cremesp-Ribeirão Preto; Renato Gregório, mestre em Administração e Desenvolvimento Empresarial; Fernanda Maris Peria, docente do Departamento de Clínica Médica da FMRP-USP; e Hermes, docente da Disciplina de Ética Médica da FMRP-USP.

\section{REFERÊNCIAS}

1. Figueiredo JF, Troncon LE, Rodrigues ML, Cianflone AR, Colares MF, Peres LC, Piccinato CE. Effect of curriculum reform on graduating student performance. Med Teach. 2004; 26(3):244-9.

2. Gonçalves EL. Trends in the graduation of Brazilian physicians. Rev Assoc Med Bras. 1996; 42(3):162-6.
3. Gonçalves EL. Medicine in Brazil today: education and practice. Rev Hosp Clin Fac Med. 1990;45(4):190-3.

4. Goncalves EL. Performance of FMUSP graduate students and curricular disciplines evaluation: in search of correlations. Rev Hosp Clin Fac Med.1993; 48(4):199-203.

5. Bueno RRL, Pieruccini MC. Abertura de escolas médicas no Brasil: relatório de um cenário sombrio. CFM. 2005; 3-67.

6. Bevilacqua RG. Medical residency and residency in medical schools. Rev Hosp Clin Fac Med. 1983; 38(5):177-8.

7. Bevilacqua RG, Sampaio SA, Azevedo AC. Work situation of physicians graduating from medical residency programs in the State of Sao Paulo, Brazil. Rev Assoc Med Bras. 1992; 38(3):127-37.

8. Marcondes E, Fujimura I, Mascaretti LA, Vanzolini ME, Lima-Goncalves E. Curriculum evaluation program of the University of Sao Paulo Medical School--results for 1993 and a 5-year analysis. Rev Hosp Clin Fac Med. 1995; 50(3):164-7.

9. Tavares C, Maia J, Muniz M, Malta M, Magalhães B, Thomaz A. O currículo paralelo dos estudantes da terceira série do curso médico da Universidade Federal de Alagoas. Rev Bras Educ Med. 2007; 31(3):245-53.

10. Drumm TL, Arkins JP, Dayan SH. Strategies to grow your practice in a struggling economy. Facial Plast Surg. 2010; 26(1):26-33.

11. Gregório R. Marketing médico. In: Garcia B, editor. Marketing médico: criando valor para o paciente. 1 . ed. Rio de Janeiro: Editora DOC; 2009. p. 17-34.

12. Nascimento AC. Medication advertising in Brazil. Can it be regulated? Cien Saude Colet. 2009;14(3):869-77.

13. Pazin-Filho A, Scarpelini S, Schmidt A. Análise qualitativa da elaboração e apresentação de aulas teóricas por alunos de pós-graduação da FMRP-USP. Medicina (Ribeirão Preto). 2007;40(1):51-62.

14. Conselho Federal de Medicina. Estabelece os critérios norteadores da propaganda em Medicina, conceituando os anúncios, a divulgaçãode assuntos médicos, o sensacionalismo, a autopromoção e as proibições referentes à matéria. Resolução nำ1.701; 2003.

15. Stata Statistical Software [computer program]. Version 10.0. College Station, TX: StataCorp; 2009.

16. Pazin-Filho A. Características do aprendizado do adulto. Medicina (Ribeirão Preto). 2007;40(1):7-16.

17. Pazin-Filho A. Aula teórica: quando utilizar? Medicina (Ribeirão Preto). 2007; 40(1):3-6.

18. Schmidt A, Pazin-Filho A. Recursos visuais. Medicina (Ribeirão Preto). 2007;40(1):32-41. 
19. Pazin-Filho A. Simpósio didática — simulação. Medicina (Ribeirão Preto). 2007;40(2):151.

20. Troncon LEA, Maffei CML. A incorporação de recursos de simulação no curso de graduação em medicina da Faculdade de Medicina de Ribeirão Preto - USP. Medicina (Ribeirão Preto). 2007;40(2):153-61. (p.8)

\section{CONTRIBUIÇÃO DOS AUTORES}

Todos os autores participaram igualmente no levantamento de dados, elaboração e aplicação do questionário, interpretação dos resultados e construção do artigo.
CONFLITO DE INTERESSES

Declarou não haver.

\section{ENDEREÇO PARA CORRESPONDÊNCIA}

Antonio Pazin-Filho

Rua Bernardino de Campus, 1000

Ribeirão Preto

CEP 14010-030 — SP

E-mail: apazin@fmrp.usp.br 